\title{
Update on Ghanem's New Scientific Discoveries in physics, Physiology, and Medicine
}

\author{
Ahmed N Ghanem* \\ El-Mansoura University, Faculty of Medicine, Retired Consultant Urologist Surgeon \& Independent Investigator, No1 President Mubarak \\ Street, Mansoura, Egypt \\ Corresponding author: Ahmed N Ghanem, El-Mansoura University, Faculty of Medicine, Retired Consultant Urologist \\ Surgeon \& Independent Investigator, No1 President Mubarak Street, Mansoura 35511, Egypt
}

\begin{tabular}{|c|}
\hline ARTICLE INFO \\
\hline Received: 幽 October 22, 2020 \\
\hline Published: October 29, 2020 \\
\hline
\end{tabular}

Citation: Ahmed N Ghanem. Update on Ghanem's New Scientific Discoveries in physics, Physiology, and Medicine. Biomed J Sci \& Tech Res 31(3)-2020. BJSTR. MS.ID.005117.

Keywords: Shock; Hyponatraemia; Fluid therapy; The TURP syndrome; ARDS; LPHS; Bladder cancer

Abbreviations: HN: Hyponatraemia; ARDS: Acute Respiratory Distress Syndrome; TURP: The Transurethral Resection of the Prostate; LPHS: Loin Pain Haematuria Syndrome; IVU 7: Intravenous Urography 7 sign; POT: Porous Orifice Tube

\section{ABSTRACT}

Introduction and Objective: To report the new scientific discoveries in physics, physiology and medicine by one author.

Material and Methods: Results of my research are summarized. It is based on 2 clinical studies one prospective and the second case series on hyponatraemia (HN) of the transurethral resection of the prostate (TURP) syndrome. A physics study on porous orifice (G) tube proves Starling's law is wrong. I reported prospective study on nephroptosis revealing its link with the loin pain haematuria syndrome (LPHS) and curative surgery for it.

Results: Two physics and two physiological discoveries are reported. Acute HN presents as shock during surgery. It is induced by massive gain of sodium-free fluid recognized as volumetric overload shock (VOS). Features of the multiple organ dysfunction syndrome occur, include ARDS, Acute renal failure (ARF) and Coma. The prospective study demonstrated volumetric overload is the most significant in patho-aetiology. The case series demonstrated mistaking VOS for a known shock and treating it with further volume expansion cause death. Correct diagnoses as VOS and treating it with hypertonic sodium is lifesaving. The physics study on the G tube demonstrated that proximal, akin to arterial, pressure induces suction not filtration producing the hydrodynamic phenomenon that replaces Starling's law. The link of LPHS with nephroptosis is demonstrated by the IVU 7 sign. The curative surgery for LPHS is renal sympathetic denervation and nephropexy.

Conclusion: Dilution HN presents as shock that is mistaken for known shocks and treated with volume expansion causing death or ARDS. Manifestations include shock, ARDS, ARF and Coma. The correct treatment is hypertonic sodium. Starling s law has proved wrong. The correct replacement is the hydrodynamics of G tube. The puzzle of LPHS was also resolved.

\section{Introduction}

This article summarizes the full list of recent scientific discoveries in physics, physiology and medicine made by a single scientific medical investigator and independent researcher who was fully self-financed supported by a full list of reported articles in reputable open access journals.

The discoveries are:

\section{Physics Discoveries}

1. The hydrodynamics of the porous orifice $(G)$ tube [1-4]

2. The Tree Branching Law (TBL) [5-8].

\section{Physiological Discoveries}

1. Proving Starling's law for the capillary-interstitial (ISF) fluid is wrong and providing the correct replacement of the magnetic field like fluid hydrodynamics of the G tube.

2. The TBL Corrects two misconceptions on capillary physiology namely:

a. The cross-section areas of all the capillaries is larger than the aorta 
b. The red blood cells (RBCs) speed in a capillary is thought "very slow" to allow for the slow perfusion of the capillary-ISF transfer as based on Starling's forces.

\section{Biochemical Discoveries}

1. Resolving the puzzle of acute dilutional hyponatraemia identifying its path-aetiology and finding a successful curative lifesaving therapy for it: The Hypertonic Sodium Therapy (HST) of $5 \% \mathrm{NaCl}$ and/or $8.4 \% \mathrm{NaCo} 3$ [9-13].

2. Revealing the effects of volume kinetics on the cardiovascular system

\section{Medical Discoveries}

1. Discovering two new types of cardiovascular shocks: the volume kinetic shocks or the volumetric overload shocks (VOS) of type one induced by sodium-free fluid and type 2 induced by sodium-based fluid retention $[14,15]$.

2. Resolving the puzzle of the acute respiratory distress syndrome (ARDS) by identifying its exact patho-aetiology being caused by VOS and a successful therapy of HST.

3. Resolving the puzzle of the transurethral resection of the prostate syndrome discovering its link with ARDS and finding the successful lifesaving therapy for it similar to that of acute hyponatraemia.

4. In discovering the above the bridge connecting the physics, physiology, biochemistry, and medicine was constructed.

5. On a totally different subject, the patho aetiology of the loin pain haematuria syndrome was discovered revealing its link with SN, and 100\% curative therapy surgery was devised.

6. A new surgical procedure for the therapy of cancer bladded with orthotopic bladder replacement was reported.

Despite multiple and powerful reporting in the literature on my multiple and important scientific discoveries the whole medical world is not responding. It seems to be in a deep coma. Even the top Medical, surgical and scientific journals including Nature, Nature Medicine, Science, Lancet, British Medical Journal, New England Journal of Medicine. Journal of The American Medical Association, The Surgeon- The Journal of the Royal College of Surgeons of Edinburgh, Physiology and Urology journals have repeatedly done serious mistakes rejecting the many articles I sent to them. They may ignore my person, but they cannot wrong any of my new discoveries. Here is a summary of my new discoveries to show you how wrong they all are [16-19].

My scientific discoveries are many and most important made over the last 32 years of my career life spent in investigating and reporting these articles. The articles recognize 2 new types of shocks and its treatment, proves that Starling's law for the capillary interstitial fluid transfer is wrong and provides an alternative mechanism: The hydrodynamics of a porous orifice (G) Tube.
These discoveries resolve the puzzles of 3 syndromes discovering its patho-aetiology and new successful treatments; namely the transurethral resection of the prostate (TURP) syndrome and acute dilution hyponatraemia $(\mathrm{HN})$, the acute respiratory distress syndrome (ARDS) and the loin pain haematuria syndrome (LPHS). Not only the exact patho-aetiologies of these syndromes were discovered but also successful treatments for it were found. The two new types of vascular shocks are volume kinetic shocks or VOS defined here.

Massive fluid infusions in a short time induce VO) of two types: Type one (VOS1) and Type two (VOS2). VOS1 is induced by sodiumfree fluid of 3.5-5 liters in one hour known as the TURP syndrome5 or hyponatraemic shock. VOS2 may complicate VOS1 or is induced by massive infusion of sodium-based fluids. VOS2 also complicates fluid therapy in critically ill and presents with ARDS.6 Volumetric gain of 12-14 litres of sodium-based fluids reported in ARDS.

Two clinical studies to understand the TURP syndrome and recognise VOS were conducted. A prospective study on 100 consecutive TURP patients of whom ten suffered TURP syndrome [5]. Volumetric overload was the only significant factor in causing the condition (Table 1 \& Figure 1). The second study was case series of 23 case cases $s$ of the TURP syndrome manifesting as VOS1. Volumetric overload quantity and type is shown in (Figure 2). Three patients died and remaining 20 patients were correctly diagnosed as VOS1 and treated with hypertonic sodium therapy (HST). Each patient passed 4-5 litres of urine followed by recovery from shock and coma. This treatment was successful in curing all patients bringing them back from dead $[19,20]$.

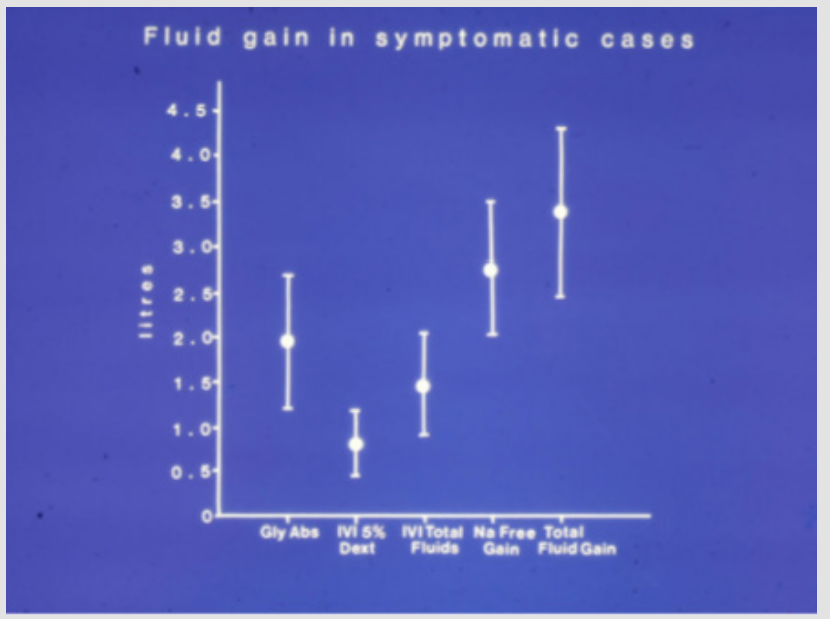

Figure 1: Shows the means and standard deviations of volumetric overload in 10 symptomatic patients presenting with shock and hyponatraemia among 100 consecutive patients during a prospective study on transurethral resection of the prostate. The fluids were of Glycine absorbed (Gly abs), intravenously infused 5\% Dextrose (IVI Dext) Total IVI fluids, Total Sodium-free fluid gained (Na Free Gain) and total fluid gain in liters. 


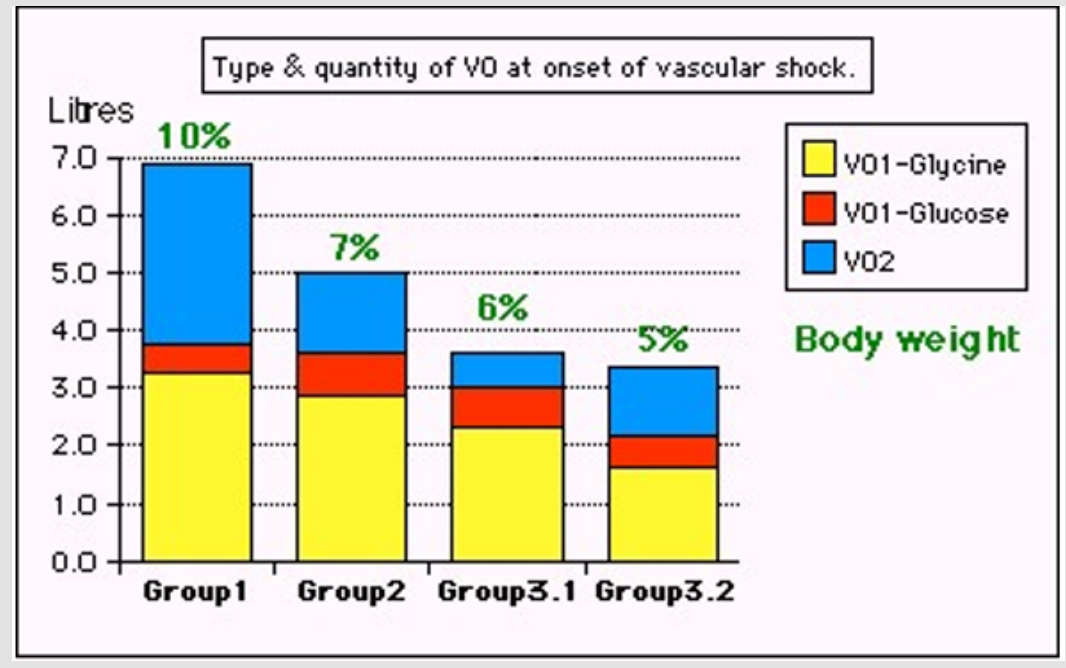

Figure 2: Shows volumetric overload (VO) quantity (in litres and as percent of body weight) and types of fluids. Group 1 was the 3 patients who died in the case series as they were misdiagnosed as one of the previously known shocks and treated with further volume expansion. Group 2 were 10 patients from the series who were correctly diagnosed as volumetric overload shock and treated with hypertonic sodium therapy (HST). Group 3 were 10 patients who were seen in the prospective study and subdivided into 2 groups; Group 3.1 of 5 patients treated with HST and Group 3.2 of 5 patients who were treated with guarded volume expansion using isotonic saline.

Table 1: Shows the multiple regression analysis of total peroperative fluid gain, drop in measured serum osmolality (OsmM), sodium, albumin, $\mathrm{Hb}$ and increase in serum glycine occurring immediately post-operatively in relation to signs of the TURP syndrome. Volumetric gain and hypoosmolality are the only significant factors

\begin{tabular}{|c|c|c|c|c|c|}
\hline Parameter & Value & Std. Err & Std. Value & T Value & P \\
\hline Intercept & & & 0.773 & & \\
\hline Fluid Gain (1) & 0.847 & 0.228 & 1.044 & 3.721 & 0.0001 \\
\hline Osmolality & 0.033 & 0.014 & -0.375 & 2.42 & 0.0212 \\
\hline Na+ (C_B) & 0.095 & 0.049 & 0.616 & 1.95 & 0.0597 \\
\hline Alb (C_B) & 0.062 & 0.087 & 0.239 & 0.713 & 0.4809 \\
\hline Hb (C_B) & -0.282 & 0.246 & -0.368 & 1.149 & 0.2587 \\
\hline Glycine (C_B) & $-4.97 \mathrm{E}-05$ & $\begin{array}{c}5.98 \mathrm{E}- \\
05\end{array}$ & -0.242 & 0.832 & 0.4112 \\
\hline
\end{tabular}

A study of the hydrodynamics of the porous orifice $(G)$ tube comparing it to that of Poiseuille's tube was done. Measurements of pressures at various parts of a circulatory system incorporating the $G$ tube in a chamber to mimic the capillary-interstitial fluid compartment were done. The effect of changing the proximal (arterial), the distal (venous) pressures and the diameter of the inlet on side pressure of the $G$ tube and chamber pressure as well as the dynamic magnetic field like fluid circulation around the $\mathrm{G}$ tube was evaluated. The dynamic magnetic field like fluid circulation around the $G$ tube and surrounding it in $C$ chamber (Figure 3) provides adequate replacement for Starling's law. The physiological equivalent of this physics study was done on the hind limbs of sheep. It demonstrated that both saline and plasma induces oedema when run through the vein not the artery, and the arterial pressure causes suction not filtration due to effect of pre- capillary sphincter.

Starling's hypothesis was based on Poiseuille work on strait uniform brass tubes. Eight decades latter evidence demonstrated that the capillary is a porous narrow orifice $(G)$ tube as it has a precapillary sphincter [8] and pores that allow the passage of plasma proteins [9]. As the capillary pores allow the passage of plasma molecules, nullifying the osmotic pressure of plasma proteins, a call for reconsideration of Starling's hypothesis was previously made [10] but there was no alternative then. The replacement came to light when the hydrodynamics of the $\mathrm{G}$ tube were discovered and reported in 2001.

The hydrodynamics of the G tube $[1,11]$ (Figure 3) demonstrated that the proximal (arterial) pressure induces a negative side pressure gradient on the wall of the $G$ tube causing suction most prominent over the proximal half and turns into positive pressure over the distal half. Incorporating the G tube in a chamber (C), representing the ISF space surrounding a capillary, demonstrated a rapid dynamic magnetic field-like fluid circulation between $\mathrm{C}$ and $\mathrm{G}$ tube lumen. Incorporating the $\mathrm{G}$ tube and $\mathrm{C}$ in a circulatory model driven by electric pump induced proximal pressure similar to arterial pressure, causing suction from $C$ into the lumen of $G$ tube. This proves that the arterial pressure causes suction not filtration at the capillary interstitial fluid circulation, and hence Starling's law is wrong on both forces and equations. The hydrodynamics of the $\mathrm{G}$ tube provide adequate correct replacement for Starling's law. This illustrates how 2 new types of vascular shocks and a replacement of Starling's law were discovered that have resolved the puzzles of 3 clinical syndromes of TURP, hyponatraemia and ARDS [21,22]. 


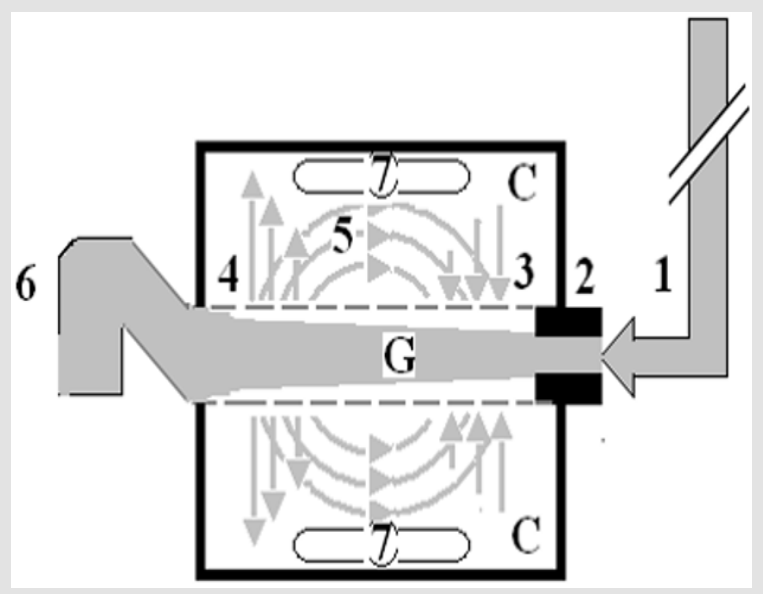

Figure 3: Shows a diagrammatic representation of the hydrodynamic of $\mathrm{G}$ tube based on $\mathrm{G}$ tubes and chamber $\mathrm{C}$. This $37-\mathrm{years}$ old diagrammatic representation of the hydrodynamic of $G$ tube in chamber $C$ is based on several photographs. The $G$ tube is the plastic tube with narrow inlet and pores in its wall built on a scale to capillary ultra-structure of precapillary sphincter and wide inter cellular cleft pores, and the chamber $C$ around it is another bigger plastic tube to form the G-C apparatus. The chamber $C$ represents the ISF space. The diagram represents a capillary-ISF unit that should replace Starling's law in every future physiology, medical and surgical textbooks, and added to chapters on hydrodynamics in physics textbooks. The numbers should read as follows:

1. The inflow pressure pushes fluid through the orifice

2. Creating fluid jet in the lumen of the $G$ tube ${ }^{* *}$.

3. The fluid jet creates negative side pressure gradient causing suction maximal over the proximal part of the $G$ tube near the inlet that sucks fluid into lumen.

4. The side pressure gradient turns positive pushing fluid out of lumen over the distal part maximally near the outlet.

5. Thus, the fluid around $\mathrm{G}$ tube inside $\mathrm{C}$ moves in magnetic field-like circulation [5] taking an opposite direction to lumen flow of $\mathrm{G}$ tube.

6. The inflow pressure 1 and orifice 2 induce the negative side pressure creating the dynamic G-C circulation phenomenon that is rapid, autonomous, and efficient in moving fluid and particles out from the $\mathrm{G}$ tube lumen at 4 , irrigating $\mathrm{C}$ at 5 , then sucking it back again at 3 ,

7. Maintaining net negative energy pressure inside chamber $\mathrm{C}$.

${ }^{* *}$ Note the shape of the fluid jet inside the G tube (Cone shaped), having a diameter of the inlet on right hand side and the diameter of the exit at left hand side (G tube diameter). I lost the photo on which the fluid jet was drawn, using tea leaves of fine and coarse sizes that runs in the centre of $G$ tube leaving the outer zone near the wall of $G$ tube clear. This may explain the finding in real capillary of the protein-free (and erythrocyte-free) sub-endothelial zone in the Glycocalyx paradigm (Woodcock and Woodcock 2012) [3]. It was also noted that fine tea leaves exit the distal pores in small amount maintaining a higher concentration in the circulatory system than that in the C chamber- akin to plasma proteins.

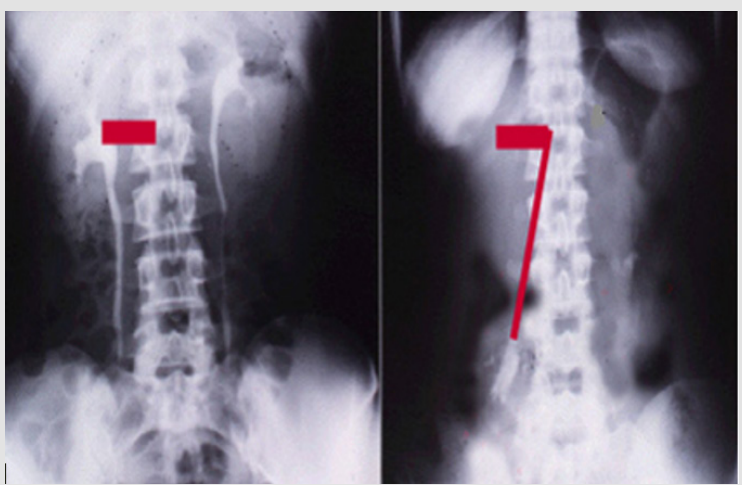

Figure 4: Shows renal pedicle mapped on a supine IVU film (Horizontal) and erect film (Vertical) limbs of 7 where the renal pedicle is stretched to 3 times its normal length, causing stenosis and ischemia.
The TBL is a fundamental law of nature that govern the ramifications of all tress of green and red of the Aorta-arterial trees. It corrects two important misconceptions on the capillary physiology. This evidence sums up to demonstrate that the capillary-ISF transfer occurs according to a precise fast circulation of the magnetic field like fluid not the slow perfusion. That provides adequately for the demands of cells at rest and increased demand during strenuous physical activity. On another subject, this article12 reports the overlooked link of Loin Pain Haematuria Syndrome with Symptomatic Nephroptosis and the Results of a new curative surgery; Renal Sympathetic Denervation and Nephropexy Surgery. Two new signs namely; the IVU 7 sign (Figure 4) and tube stretch hypothesis were reported demonstrating that renal pedicle stretch causing vessel stenosis, ischaemia and neuropathy. Surgical treatment was used in 28 patients; 10 had simple nephropexy and 18 had Renal 
Sympathetic Denervation and Nephropexy Surgery (RSD\&N) for severe LPHS. Four of patients treated with simple nephropexy had recurrence of LPHS while those who had RSD\&N were all cured.

On another subject I reported a surgical point of technique [13] for operable cancer bladder in which "capsule sparing" cystoprostadenectomy for orthotopic bladder replacement that overcomes the problems of difficult urethral anastomosis, impotence and incontinence.

\section{Conclusion}

Two new physics discoveries of the G tube hydrodynamics and tree branching law with two related physiological discoveries of proving Starling's law wrong and correcting two misconceptions on capillary physiology, and 6 new medical discoveries are reported. These resolved the puzzles of dilution HN of the TURP syndrome that presents as shock mistaken for known shocks and treated with volume expansion causing death or ARDS. Manifestations include shock, ARDS, ARF and Coma. The correct treatment is hypertonic sodium therapy. Starling's law has proved wrong. The correct replacement is the hydrodynamics of $\mathrm{G}$ tube. The puzzle of LPHS was also resolved. A new point of technique for bladder replacement was reported.

\section{References}

1. Ghanem AN, Ghanem SA (2016) Volumetric Overload Shocks: Why Is Starling's Law for Capillary Interstitial Fluid Transfer Wrong? The Hydrodynamics of a Porous Orifice Tube as Alternative. Surg Sci 7(6): 245-249.

2. Pindoria N, Ghanem SA, Ghanem KA, Ghanem AN (2017) Volumetric overload shocks in the patho-etiology of the transurethral resection prostatectomy syndrome and acute dilution hyponatraemia. Integr Mol Med 4: 1000279.

3. Ghanem KA, Ghanem AN (2017) The proof and reasons that Starling's law for the capillary-interstitial fluid transfer is wrong, advancing the hydrodynamics of a porous orifice $(G)$ tube as the real mechanism. Blood, Heart and Circ Volume 1(1): 1-7.

4. Ghanem KA, Ghanem AN (2017) Volumetric overload shocks in the patho-etiology of the transurethral resection prostatectomy syndrome and acute dilution hyponatraemia: The clinical evidence based on 23 case series. Basic Res J Med Clin Sci 6(4): 35-43.

5. Ghanem AN (2019) The Tree Branching Law: Correcting misconceptions on capillary cross-section areas and blood speed.

\section{ISSN: 2574-1241}

DOI: 10.26717/BJSTR.2020.31.005117

Ahmed N Ghanem. Biomed J Sci \& Tech Res

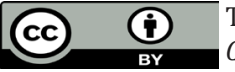

This work is licensed under Creative

Commons Attribution 4.0 License

Submission Link: https://biomedres.us/submit-manuscript.php
6. Ghanem AN (2020) Capillary ultrastructure anatomy and physiology: what is known, what is unknown or missing, what is wrong, and what is new?.

7. Ghanem AN (2018) Final proof Starling's law wrong and G tube correct replacement: New results and critical analytical criticisms of landmark articles.

8. Ghanem AN (2020) New Physics Discoveries of Relevance to Capillary Physiology and Clinical Significance to Newly Recognized Volumetric Overload Shocks.

9. Ghanem AN, Ward JP (1990) Osmotic and metabolic sequelae of volumetric overload in relation to the TURP syndrome. Br J Uro 66(1): $71-78$

10. Ghanem AN (2019) Post-Surgical Hyponatraemia: Problems of Management Resolved by Revealing its Relation to Volumetric Overload Shocks. EC Cardiology 6(8).

11. Ghanem AN (2019) Postoperative Dilution Hyponatraemia and the TURP Syndrome: Critical Analytical Review of Literature on PathoEtiology and Therapy. EC Emerg Med Crit Care 3(8): 507-514.

12. Ghanem AN (2018) Hyponatraemia: Nadirs and Paradoxes of the Missing Volumetric Overload. Open Access J Surg 10(2): 555781.

13. Ghanem AN (2018) Therapy of Hyponatremia: End of Era or Minority Report? Biomed J Sci \& Tech Res 11(4): 8645-8648.

14. Ghanem (2020) Volumetric Overload Shocks (VOS) in Surgical Patients. Open Access J Surg 11(2): 555810.

15. Ashbaugh DG, Bigelow DB, Petty TL, Levine BE (1967) Acute respiratory distress in adults. Lancet 2(7511): 319-323.

16. Starling EH (1886) Factors involved in the causation of dropsy. Lancet 2: $1266-1410$

17. Rhodin JA (1967) The ultra-structure of mammalian arterioles and precapillary sphincters. J Ultrastructure Res 18(1-2): 181-222.

18. Karnovesky MJ (1967) The ultra structural basis of capillary permeability studied with peroxidase as a tracer. J Cell Biol 35(1): 213-236.

19. Renkin EM (1986) Some consequences of capillary permeability to macromolecules: Starling's hypothesis reconsidered. Am J Physiol (Heart Circ Physiol) 250(5 Pt 2): H706-H710.

20. Ghanem AN (2001) Magnetic field-like fluid circulation of a porous orifice tube and relevance to the capillary-interstitial fluid circulation: Preliminary report. Medical Hypotheses 56(3): 325-334.

21. Ghanem AN (2016) Prospective Observational Study on Loin Pain Hematuria Syndrome Complicating Symptomatic Nephroptosis and the Results of Renal Sympathetic Denervation and Nephropexy Surgery. J J Nephro Urol 3(1): 024

22. Ghanem AN (2002) Experience with "capsule sparing" cystoprostadenectomy for orthotopic bladder replacement: Overcoming the problems of impotence, incontinence and urethral anastomosis. BJU Int 90: 617-620

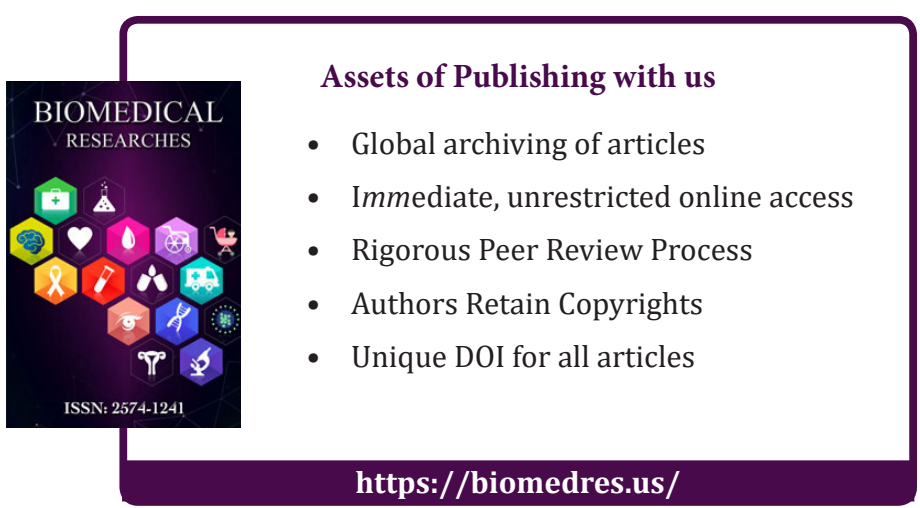

Copyright@ Ahmed N Ghanem | Biomed J Sci \& Tech Res | BJSTR. MS.ID.005117. 